\title{
Wolbachia, sex ratio bias and apparent male killing in the harlequin beetle riding pseudoscorpion
}

\author{
DW Zeh ${ }^{1}$, JA Zeh ${ }^{1}$ and MM Bonilla ${ }^{2}$ \\ ${ }^{1}$ Department of Biology and Program in Ecology, Evolution and Conservation Biology, University of Nevada, Reno, NV 89557, USA; \\ ${ }^{2}$ Department of Biochemistry, University of Nevada, Reno, NV 89557, USA
}

\begin{abstract}
Bacterial endosymbionts that manipulate host reproduction are now known to be widespread in insects and other arthropods. Since they inhabit the cytoplasm and are maternally inherited, these microorganisms can enhance their fitness by biasing host sex ratio in favour of females. At its most extreme, sex ratio manipulation may be achieved by killing male embryos, as occurs in a number of insect species. Here, we provide evidence for the first case of male killing by a tetracycline-sensitive microbe in pseudoscorpions. Using a combination of inheritance studies, antibiotic treatment and molecular assays, we show that a new strain of Wolbachia is associated with extreme female bias in the pseudoscorpion, Cordylochernes scorpioides. In a highly female-biased line, sex ratio distortion was maternally inherited, and occurred in conjunction with a high rate of spontaneous abortion and low reproductive success. Anti-
\end{abstract}

biotic treatment cured females of the Wolbachia infection, restored offspring sex ratio to $1: 1$, and significantly enhanced female reproductive success. The discovery of apparent male-killing in $C$. scorpioides is of interest because pseudoscorpions are viviparous. Theory predicts that male killing should be favoured, if male death enhances the fitness of infected female siblings. In a live-bearing host, reallocation of maternal resources from dead male embryos to their sisters provides a direct, physiological mechanism through which fitness compensation could favour male killing by cellular endosymbionts. Our results suggest, however, that fitness compensation and the spread of male-killing endosymbionts may be undermined by a high rate of spontaneous abortion in infected females of this viviparous arthropod.

Heredity (2005) 95, 41-49. doi:10.1038/sj.hdy.6800666

Published online 1 June 2005

Keywords: Cordylochernes scorpioides; male killing; selfish genetic elements; sex ratio distortion; viviparity; Wolbachia

\section{Introduction}

As a consequence of their maternal mode of inheritance, genetic elements in the cytoplasm can increase their fitness by biasing sex ratio in favour of females (Eberhard, 1980; Cosmides and Tooby, 1981). Such sex ratio distortion is known to be widespread in invertebrates (Majerus, 2003) and angiosperm plants (Budar et al, 2003), and is achieved through a variety of mechanisms by a diversity of cytoplasmic agents. For example, various bacteria, including Arsenophonus, Flavobacteria, Rickettsia and Spiroplasma, have been implicated in the killing of male embryos in Nasonia wasps (Gherna et al, 1991), ladybird beetles (Werren et al, 1994; Hurst et al, 1999a; Schulenburg et al, 2002), buprestid beetles (Lawson et al, 2001), butterflies (Jiggins et al, 2000) and fruit flies (Williamson and Poulson, 1979). Among eukaryotic microorganisms, intracellular parasites in the phylum Microsporidia are known to engage in the killing and/or feminization of immature males in both insects and crustaceans (Dunn and Smith, 2001).

To date, the most intensively studied sex ratio distorting organisms are members of the bacterial lineage, Wolbachia. Estimates of the percentage of insect

Correspondence: DW Zeh, Department of Biology and Program in Ecology, Evolution and Conservation Biology, University of Nevada, Mail Stop 314, Reno, NV 89557, USA. E-mail: zehd@unr.edu

Received 4 April 2004; accepted 14 February 2005; published online 1 June 2005 species infected with these obligately, intracellular bacteria range from $16 \%$ (Werren et al, 1995) to $76 \%$ (Jeyaprakash and Hoy, 2000), and Wolbachia has also been isolated from nematodes, amphipods, isopods, mites and spiders (Werren, 1997; Charlat et al, 2003; Rowley et al, 2004). As a group, Wolbachia exhibit great versatility in their strategies for achieving female bias. They may feminize males of diploid species, as in isopods and amphipods (Bouchon et al, 1998). They may induce parthenogenesis in haplodiploid species, by converting haploid males into diploid females (Stouthamer and Kazmer, 1994). Finally, Wolbachia may manipulate sex ratio by killing male embryos early in development, as occurs in some species of beetles (Hurst et al, 1999b; Fialho and Stevens, 2000), butterflies (Hurst et al, 1999b; Dyson et al, 2002), moths (Kageyama et al, 2003) and fruit flies (Hurst et al, 2000).

Here, we report the first apparent case of male killing by a tetracycline-sensitive microbe in the arachnid order, Pseudoscorpionida. Using a combination of inheritance studies, antibiotic treatment and molecular assays, we show that a previously undescribed strain of Wolbachia is associated with extreme female bias and low reproductive success in the harlequin beetle riding pseudoscorpion, Cordylochernes scorpioides. This pseudoscorpion has already proved to be an excellent model system for laboratory and field investigations of genetic incompatibility (Newcomer et al, 1999), sexual selection (Zeh et al, 1997; 1998) and speciation (Zeh and Zeh, 1994; Zeh et al, 2003). Laboratory rearing of the progeny of field- 
collected, C. scorpioides females reveals extensive variation in offspring sex ratio, with a small proportion of females producing highly female-biased broods.

Unlike the vast majority of terrestrial arthropods (Zeh and Smith, 1985), pseudoscorpions are viviparous, with embryos developing in a translucent brood sac, overlying the female's genital aperture (Weygoldt, 1969). Using a specialized mouth precursor (the pumping organ), individual embryos actively draw nutritive fluid, consisting of phospholipids, proteins and polysaccharides (Makioka, 1968), from the mother's reproductive tract (Weygoldt, 1969). This 'external-womb' form of viviparity (Zeh and Zeh, 2000) greatly facilitates visual assessment of the postfertilization consequences of endosymbiont infection for embryonic development in a live-bearing species.

\section{Materials and methods}

\section{Sex ratio survey}

Pseudoscorpions were collected as nymphs from large populations inhabiting decaying fig trees (Ficus spp.) in the former Canal Zone $(\mathrm{CZ})$ and surrounding areas of the Republic of Panama (see Zeh et al, 2003). To provide virgin females for the study, nymphs were maintained individually in vials in the laboratory until they molted to the adult stage. These field-collected, virgin females were each permitted to mate with a single male. In order to determine brood sex ratio, their progeny were then reared individually to the adult stage, since male and female pseudoscorpions are not distinguishable as immatures. Only females producing 10 or more adult offspring $(n=153)$ were included in the sex ratio survey.

\section{Establishment of female-biased (FB) isofemale line}

Following the sex ratio survey, an isofemale line was established from the progeny of a female collected in the area of Llano Cartí $\left(09^{\circ} 18^{\prime} \mathrm{N}, 78^{\circ} 58^{\prime} \mathrm{W}\right.$; see Zeh et al, 2003). This female produced only daughters (17 females and zero males). To investigate inheritance of the sex ratio bias, the $17 \mathrm{~F}_{1}$ females were mated to unrelated males from families with sex ratios not significantly different from 1:1. The resultant $F_{2}$ male and female offspring were similarly outcrossed to produce an $\mathrm{F}_{3}$ generation.

\section{Tetracycline treatment experiment}

To determine the effect of antibiotic (tetracycline) treatment on female reproductive success and offspring sex ratio in the FB line, we performed a split-brood experiment in which the offspring produced by each of 10 FB-line females were randomly assigned at birth, that is, as protonymphs, to either an untreated (control) or a tetracycline-treated rearing regime. Protonymphs were reared at $28.5^{\circ} \mathrm{C}$ to the adult stage in individual vials and were provisioned weekly with Drosophila melanogaster larvae. After the final nymphal molt to the adult stage, these offspring were switched to a diet of late-instar Tribolium confusum larvae. In the antibiotic treatment, tetracycline was administered to pseudoscorpions by rearing their prey on medium to which tetracycline hydrochloride (Sigma) was added. For D. melanogaster, 4-24 ${ }^{\mathbb{B}}$ instant Drosophila medium (Carolina Biological Supply Company) was supplemented with tetracycline at a concentration of $0.52 \mathrm{mg} / \mathrm{ml}$. For T. confusum, larvae were reared in an 8:1 (v/v) mixture of wheat flour and brewer's yeast containing a $0.2 \%$ concentration $(\mathrm{w} / \mathrm{w})$ of tetracycline hydrochloride. On reaching sexual maturity, each virgin female pseudoscorpion from the two rearing regimes was mated to a single, unrelated male, and was then monitored to determine the number of protonymphs she produced (see Zeh and Zeh, 2001). After mating, C. scorpioides females exhibit one of three possible outcomes: (1) failure to produce a brood sac; (2) production of a brood sac but spontaneous abortion of the entire brood before birth of the protonymphs; or (3) brood sac production and carrying of embryos to term, with all protonymphs in the brood birthing simultaneously. To assess offspring sex ratio in the two treatments, from each female that successfully gave birth, we reared 25 randomly selected protonymphs to the adult stage in individual vials, and the remaining protonymphs in the brood were frozen at $-70^{\circ} \mathrm{C}$ for subsequent PCR screening (see below).

\section{PCR assay for the presence of Wolbachia}

Initial PCR screening of total genomic DNA, extracted from adult FB-line individuals using a $2 \times \mathrm{CTAB}$ protocol (Zeh et al, 1992), was carried out with the Wolbachia-specific ftsZ and wsp primer pairs described in Jeyaprakash and Hoy (2000, p 403). The ftsZ primers yielded weak and inconsistent amplification products. Gel-isolated bands were therefore PCR reamplified to provide DNA sequence from which an fts $\mathrm{Z}$ primer pair was redesigned for $C$. scorpioides, as follows: GGACTT GAAGAACTGCAAAAATACG (CsftsZ-F) and TTGAT CAAAGGTAGCACCAAATATTATG (CsftsZ-R). In order to increase yield and reduce primer/dimer artifacts, the wsp primers were also redesigned, as follows: GGTCCAATAAGTGATGAAGAAACTAGC (Cswsp-F) and TTAAACGCTACTCCAGCTTCTGC (Cswsp-R). These redesigned primers were then used in two, separate PCR assays. First, to provide a preliminary estimate of the prevalence of Wolbachia infection in this pseudoscorpion, PCR screening was carried out on a random sample of $C$. scorpioides adults derived from field populations in two locations in central Panama (Camino Madden (MAD) and Llano Cartí (LC); see Zeh et al, 2003), as well as on individuals spanning multiple generations of the FB-line. Second, to confirm the effectiveness of the antibiotic treatment, protonymphs produced by untreated and tetracycline-treated females were individually PCR-screened for the presence/ absence of Wolbachia. As a sample of protonymphs from each of these broods had been reared to sexual maturity for sex ratio assessment, we were also able to investigate the relationship between brood sex ratio and proportion of protonymphs testing positive for Wolbachia.

PCR reactions were carried out in a $25 \mu \mathrm{l}$ volume containing $50 \mathrm{mM} \mathrm{KCl}, 10 \mathrm{mM}$ Tris- $\mathrm{HCl}$ (pH 9.0), $0.1 \%$ Triton X-100, $2.75 \mathrm{mM} \mathrm{MgCl}_{2}, 0.1 \mathrm{mM}$ dNTPs and $0.5 \mathrm{U}$ Titanium Taq DNA polymerase. PCR amplification conditions involved an initial 2 min melting step at $94^{\circ} \mathrm{C}$, followed by 30 iterations of the following cycle: $94^{\circ} \mathrm{C}$ for $40 \mathrm{~s}, 60^{\circ} \mathrm{C}$ for $60 \mathrm{~s}$, and $68^{\circ} \mathrm{C}$ for $60 \mathrm{~s}$, with a final 7 -min extension at $68^{\circ} \mathrm{C}$. For PCRs involving adults, approximately $10 \mathrm{ng}$ of extracted genomic DNA was included in the reaction mixture. In the case of protonymph screening, the whole (unprocessed), frozen 
individual was added to the reaction mixture. The suitability of DNA templates for PCR was verified by carrying out a reaction to amplify the cCsMS23 minisatellite DNA locus, originally isolated from a genomic library of C. scorpioides (Zeh et al, 1994). Protonymphs invariably remain intact after a PCR reaction. After testing for Wolbachia, it was therefore possible to carry out second PCR reactions on the same protonymphs by pipetting off the original PCR solution and adding new reagents. Samples that failed to amplify were excluded from the Wolbachia PCR screen.

Several precautions were taken to avoid sample contamination. We used aerosol-resistant pipette tips, prepared small aliquots of reagent stocks, and exposed PCR reactions to $150 \mathrm{~mJ}$ of UV radiation to crosslink any contaminant DNA before adding template DNA. To avoid possible gut-content contamination of the pseudoscorpion samples, all adult C. scorpioides were starved for a minimum of 5 days before being frozen for PCR assays. This did not apply to protonymphs, since they were frozen immediately after birth. In addition, we tested for Wolbachia infection in our laboratory cultures of D. melanogaster and T. confusum. For this assay, we performed PCR on early-instar larvae of the two prey species, using the redesigned wsp primers.

To test for the possible presence of non-Wolbachia, endosymbiotic bacteria in the FB-line, we carried out PCR and sequencing of 10 FB-line individuals, using the highly conserved, $16 \mathrm{~S}$ rDNA primers, fD1 and rP2 (Weisburg et al, 1991). These primers amplify DNA from nearly all Eubacteria, and are therefore capable of detecting infection by multiple bacterial species. For each individual, PCR yielded a single, intense band, which was then sequenced directly using the fD1 primer. The resulting sequences from all individuals were identical and extremely clean (data not shown), suggesting the presence of a single bacterial strain. A BLAST search found 96-98\% sequence homology between this sequence and more than 100 Wolbachia sequences deposited in GENBANK.

\section{DNA sequencing}

PCR templates were prepared for sequencing by electrophoresing $12 \mu \mathrm{l}$ of the reaction through a $1 \%$ agarose gel stained with ethidium bromide. Amplification products were excised from gels and purified using Promega Wizard minicolumns. BigDye reactions $(6 \mu \mathrm{l})$ containing approximately $100 \mathrm{ng}$ of purified PCR product and $3.2 \mathrm{pmol}$ of PCR primer were directly sequenced using an ABI Prizm 3730 automated sequencer, according to the manufacturer's protocols (PE Applied Biosystems). For the gut-content contamination test, PCR wsp fragments amplified from three $D$. melanogaster and three T. confusum larvae were prepared and sequenced in the forward direction, as described above.

\section{Phylogenetic analysis}

To determine the evolutionary relationship of the Wolbachia strain isolated from FB-line individuals with strains described from other arthropods, the C. scorpioides wsp sequence was incorporated into a previously published sequence alignment for 28 group $\mathrm{A}$ and $\mathrm{B}$ strains of Wolbachia deposited in the EMBL database (Accession Number DS32273; Zhou et al, 1998). As this alignment was originally produced before the discovery of male killing by Wolbachia in 1999, we also incorporated five sequences of newly-discovered male-killing Wolbachia, listed by host taxon, as follows: (1) Drosophila bifasciata (Hurst et al, 2000); (2) Adalia bipunctata strain Z and (3) strain Y (Hurst et al, 1999b); (4) Acraea encedon (Schulenburg et al, 2000), and (5) Ostrinia scapulalis (Kageyama et al, 2003). In addition, we included two sequences, Diaea circumlita c3 (Rowley et al, 2004) and Dysdera erythrina (Cordaux et al, 2001), both isolated from spiders, and belonging to a cluster tentatively identified as Wolbachia group ' $\mathrm{G}$ ' (Rowley et al, 2004). Finally, with the aim of rooting the arthropod Wolbachia tree, we included four sequences from the filarial nematode Wolbachia C and D groups, as follows: Onchocerca gutturosa (Casiraghi et al, 2001) and Dirofilaria immitis (Bazzocchi et al, 2000) from group C, and Brugia malayi and Wuchereria bancrofti (Bazzocchi et al, 2000) from group D.

Alignment of the new sequences was carried out, using both the MacDNASIS multiple sequence editor (Hitachi software, v3.7, 1997, San Bruno, CA, USA), and the data editor in MacClade, version 4.05 (Maddison and Maddison, 2002). Maximum parsimony (MP) branchand-bound and heuristic maximum likelihood (ML) searches were performed on aligned sequences, as implemented in PAUP v4.0b10 (Swofford, 2002), and all minimal trees were saved. Hierarchical likelihood ratio tests were performed using MODELTEST v3.6 (Posada and Crandall, 1998), in order to determine the best-fit ML substitution model available from the set of 56 models implemented in the program. Support for each clade was estimated by analyzing 1000 and 100 bootstrap replicate data sets, respectively, for the MP and ML searches (Felsenstein, 1985). Pairwise genetic distances between sequences were calculated using both uncorrected and ML methods.

\section{Results}

\section{Sex ratio distribution}

The 153 C. scorpioides females produced a total of 1567 female and 1487 male offspring, a ratio not significantly different from 1:1 $\left(P>0.05, \chi^{2}=2.10\right)$. However, this overall unbiased sex ratio masked extensive variation between dams, with the proportion of offspring that were males ( $\left.p_{\text {male }}\right)$ ranging from 0.00 to 0.82 (Figure 1). Whereas only one dam produced a significantly malebiased brood $\left(P<0.01, \chi^{2}\right.$ test), the broods of eight dams were significantly female biased, with the two most extremely biased families consisting of 38 females and two males $\left(P<0.0001, \chi^{2}=32.40\right)$, and 17 females and zero males $\left(P<0.0001, \chi^{2}=17.00\right)$. An isofemale line generated from this latter family is henceforth referred to as the FB line.

\section{Maternal inheritance of sex ratio bias in FB line}

In subsequent generations of the $\mathrm{FB}$ line, the sex ratio bias was found to be heritable through females but not through males. Eight outcrossed $F_{1}$ females, from which two broods per female were reared, produced a total of 328 female and 37 male offspring. A single brood of offspring reared from each of $21 \mathrm{~F}_{2}$ females yielded 275 females and 62 males. By contrast, $F_{2}$ males from the FB line $(n=18)$ sired 260 female and 234 male offspring, 
44

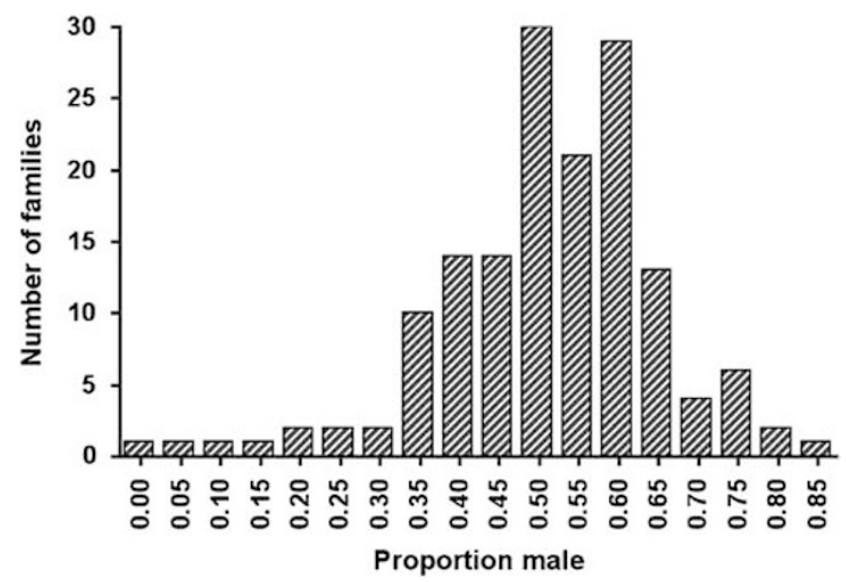

Figure 1 Sex ratio (proportion male) of laboratory-reared broods produced by 153 Cordylochernes scorpioides females that had each been mated to a single male. Only broods with 10 or more offspring are included in the histogram.

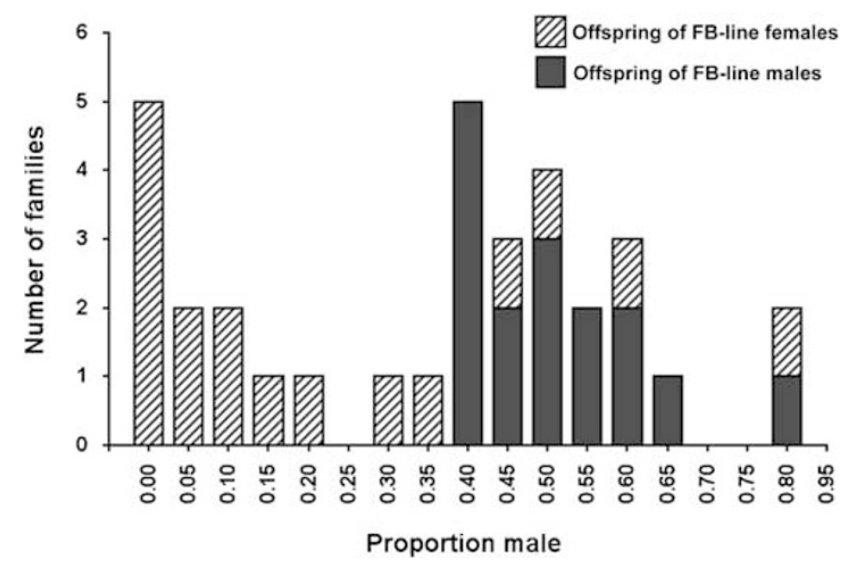

Figure 2 Sex ratio (proportion male) of offspring produced by $\mathrm{F}_{2}$ generation FB-line males and females. Only broods with 10 or more offspring are included in the histogram.

based on a single brood of offspring per male. For statistical comparison of $F_{3}$ sex ratios by lineage type (maternal or paternal), only families consisting of 10 or more adult offspring were included, and each family was treated as a replicate, with family sex ratio

$$
\left(\arcsin \left(\sqrt{p_{\text {male }}}\right)\right)
$$

as the response variable. This analysis revealed a highly significant effect of lineage type on offspring sex ratio $(P<0.0001, t=4.37$, d.f. $=31)$. The mean proportion of males $( \pm S E)$ in the maternal line was $0.20 \pm 0.06$, compared to $0.49 \pm 0.03$ in the paternal line (Figure 2).

\section{Tetracycline treatment experiment}

Treatment of FB-line dams with tetracycline increased female reproductive success and restored offspring sex ratio to $1: 1$. The treated dams $(n=39)$, gave birth to a mean $( \pm \mathrm{SE})$ of $21.44 \pm 4.67$ protonymphs compared to a mean of $6.72 \pm 2.47 \quad(n=40)$ for untreated females $(P=0.026$, Mann-Whitney $U=597.5)$. The higher reproductive success achieved by treated females was attributable both to a lower rate of spontaneous abortion of entire broods compared to untreated females (15 of 30 gravid, treated females vs 18 of 26 gravid, untreated females; $P=0.07$, Fisher exact test) and to a higher number of protonymphs produced by females that successfully gave birth (mean $\pm 90 \%$ confidence interval $(\mathrm{CI})=55.7 \pm 7.4$ for treated females, compared to $33.6 \pm 12.1$ for untreated females; $P=0.013$, Mann-Whitney $U=21.5)$. Although this difference is statistically significant, the CI were large because of the small sample size in the nontetracycline treatment. With many of the untreated FB-line females spontaneously aborting their entire broods, only eight of 40 untreated females actually gave birth to nymphs. To address this issue, we therefore included a second analysis, which incorporated data on the number of nymphs born to an additional 41 untreated FB-line females that were not part of the tetracycline treatment experiment, for a total sample size of 49 . The mean $\pm 90 \% \mathrm{CI}$ for the combined data set was $33.2 \pm 4.3(90 \%$ CI range $=28.9-37.5)$. This represents a percent brood reduction ranging from 32.7 to $48.1 \%$, and corresponds well with the expected percentage rate of brood reduction that would result from male-killing. As sex ratio distortion is not absolute (the proportion male in the FB line is approximately $10 \%$, see below), male killing should reduce the number of nymphs born by approximately $44.5 \%$.

Among the successful females, the mean proportion of males $\left(p_{\text {male }}\right)$ produced by tetracycline-treated dams was $0.51 \pm 0.036$, whereas the mean for the control dams was $0.10 \pm 0.046$. This difference was highly significant $(P=0.001$, Mann-Whitney $U=230)$. The mean number of daughters born to treated and untreated FB dams was estimated for each of the two categories of successful females by multiplying the mean number of protonymphs born by the proportion of offspring that were identified as females among the subset of nymphs $(n=25$ per brood) that were reared to sexual maturity. The resulting mean of 27.3 females born to treated FB-line dams (proportion female $=0.49$; mean number of protonymphs born $=55.7$ ) was very similar to the mean of 30.2 for untreated FB-line dams (proportion female $=0.90$; mean number of protonymphs born $=33.6$ ). Moreover, treated dams produced nearly twice the number of protonymphs born to untreated females. Taken together, these results are comparable to the 50\% reduction in hatch rate that is commonly taken as evidence of male killing in egg-laying insects (eg Fialho and Stevens, 2000). Survivorship from protonymph to the adult stage

$$
(\arcsin (\sqrt{\text { proportion surviving }}))
$$

did not differ between the two treatments $(P>0.10$, $t=1.06$; d.f. $=15$ ), indicating that the tetracycline-sensitive microbe exerts its effects only during embryonic development.

\section{PCR assay for the presence of Wolbachia}

DNA extracted from all 12 adult (six male and six female) pseudoscorpions from the MAD location tested negative when assayed with the ftsZ primers. Of the 13 adults (seven female and six male) from the LC location, two females tested positive and the remainder negative. By contrast, strong amplification products were obtained from all PCR reactions carried out on DNA extracted from 16 adult FB line individuals (10 female and six male). For molecular assessment of the effectiveness of 


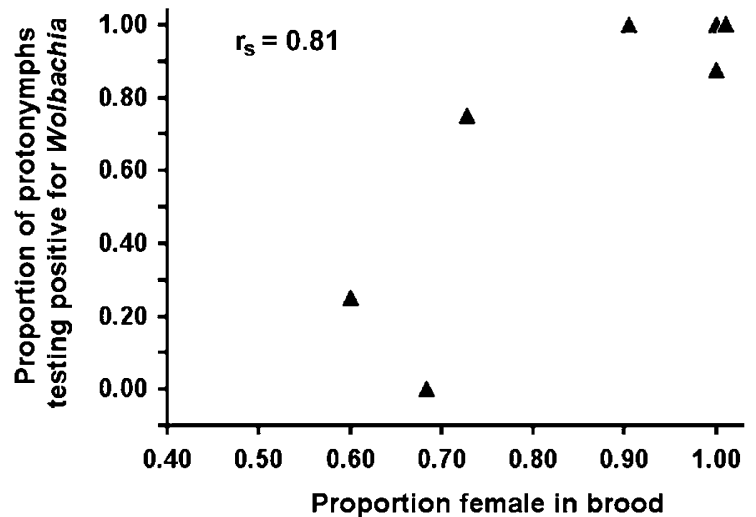

Figure 3 Bivariate plot showing the relationship between the proportion of protonymphs in an untreated FB brood that tested PCR positive for Wolbachia infection and the proportion of the brood that was female.

the antibiotic treatment, PCR was performed on 64 protonymphs produced by seven untreated and seven tetracycline-treated FB line females. None of the 28 protonymphs from treated females yielded an amplification product. Of 36 protonymphs born to seven untreated females (mean of five protonymphs per dam), 26 tested positive and 10 negative for Wolbachia. This difference was highly significant $(P<0.0001$, Fisher exact test). Moreover, in broods produced by untreated females, there was a strong, positive correlation $\left(P=0.028, r_{\mathrm{s}}=0.81\right.$, d.f. $\left.=6\right)$ between the proportion of protonymphs in a brood that tested positive for Wolbachia infection, and the proportion of the brood that was female (Figure 3).

Sequencing of wsp gene PCR products obtained from our laboratory stocks of $T$. confusum and $D$. melanogaster enabled us to reject the possibility of gut-content contamination of the pseudoscorpion samples. Both the beetle and the fly cultures were found to be infected with Wolbachia. In each case, however, the wsp sequence was identical to that of a strain previously isolated from the species (GenBank accession numbers AF020083 and AF020072 for T. confusum and D. melangaster, respectively, Zhou et al, 1998), and was highly divergent from that of the C. scorpioides Wolbachia strain.

\section{DNA sequencing}

The ftsZ and wsp gene fragments from 10 FB-line individuals were sequenced in the forward direction, yielding approximately $1000 \mathrm{bp}$ (400 bp ftsZ; $600 \mathrm{bp} \mathrm{wsp)}$ of total sequence that was identical in all individuals. Sequencing in the reverse direction for the two genes was therefore restricted to amplification products from two of these FB-line individuals. Sequencing in both directions provided nucleotide data for a $446 \mathrm{bp}$ segment of the ftsZ gene and a $628 \mathrm{bp}$ segment of the wsp gene (GenBank accession numbers AY916133 and AY916134 for wsp and fts $Z$, respectively).

\section{Phylogenetic affinities of the $C$. scorpioides Wolbachia strain}

NCBI BLAST searches conducted for both the ftsZ and wsp genes indicated that the sequences obtained from C. scorpioides show considerable divergence from any known Wolbachia sequences, with the ftsZ gene being only $92 \%$ identical (413 of $446 \mathrm{bp}$ ) to that of the strain with the highest BLAST similarity score (see Altschul et al, 1997), isolated from a species of Mellitobia (accession number U28191; Werren et al, 1995). Similarly, the wsp gene sequence from C. scorpioides is only $91 \%$ identical (334 of $364 \mathrm{bp)}$ to the sequence with the highest similarity score (strain isolated from Drosophila simulans (WHA); Accession Number AF020068; Zhou et al, 1998).

To simplify the phylogenetic analyses, identical sequences in the Zhou et al, (1998) alignment were collapsed into single, unique sequences, as follows: (1) D. melanogaster (AUB); D. melanogaster (Cairns); D. melanogaster (CS); D. melanogaster (yw); (2) D. simulans (WHA); D. sechellia; (3) Culex quinquefasciatus; C. pipiens; and (4) D. simulans (Wat/Mau); D. simulans (Noumea) (see Figure 4). The wsp sequence from $C$. scorpioides was highly divergent from all other sequences in the alignment, with uncorrected nucleotide sequence distances ranging between 13.49 and $24.05 \%$ $($ mean $=20.20 \%)$, compared to ML distances of 17.67 $43.14 \%$ (mean $=33.61 \%$ ). Closest to the C. scorpioides strain was the sequence from the spider, Dysdera erythrina. MP analysis yielded 18 most parsimonious trees (tree length $=861$, consistency index $=0.508$ ), which differed only slightly in overall topology. The single, ML tree ( $-\operatorname{Ln} L=4726.67$ ) had a length of 870 and a topology similar but not identical to that of the MP trees. Unexpectedly, in the ML analysis and some of the MP trees, the C. scorpioides Wolbachia strain was not placed in the arthropod A/B group but was inserted between the filarial nematode Wolbachia groups D and C, as the sister taxon to a clade consisting of the $\mathrm{C}+\mathrm{G}+\mathrm{A}+\mathrm{B}$ groups (Figure 4). To test for rate constancy of nucleotide substitutions across the tree, we carried out a loglikelihood ratio test using PAUP. This analysis indicated that evolutionary substitutions in wsp sequences strongly violate the assumption of a molecular clock ( $-\mathrm{Ln}$ likelihood $=5094.34$ for clock-enforced tree vs 4726.67 for nonclock tree, $P<0.001, \chi^{2}=735.34$, d.f. $=32$ ). Branch swapping performed in MacClade also indicated that support for the placement of the C. scorpioides sequence between the $\mathrm{D}$ and $\mathrm{C}$ nematode Wolbachia clades was weak: alternative placement of the $C$. scorpioides sequence as the sister group of the D clade had no effect on tree length, while inserting the sequence between the $C$ and $G$ clades involved an increase of only two steps. Moreover, a preliminary, ftsZ gene phylogenetic analysis, incorporating 17 strains from groups $\mathrm{A}$ through $\mathrm{F}$ and the $446 \mathrm{bp}$ of sequence available from the $C$. scorpioides Wolbachia strain, suggests that the $C$. scorpioides sequence may be most closely related to sequences from collembolans and termites in the Wolbachia supergroups $\mathrm{E}$ and F, respectively (see Lo et al, 2002). Unfortunately, wsp sequences are not yet available for these group $\mathrm{E}$ and $\mathrm{F}$ taxa. Again, because the $C$. scorpioides ftsZ sequence was highly divergent from other sequences (minimum ML distance $=9.87 \%$ ), and because of a high level homoplasy (consistency index $=0.569$ ), support for this placement was weak.

\section{Discussion}

The results of this study provide evidence linking a new strain of Wolbachia to extreme female bias in the 


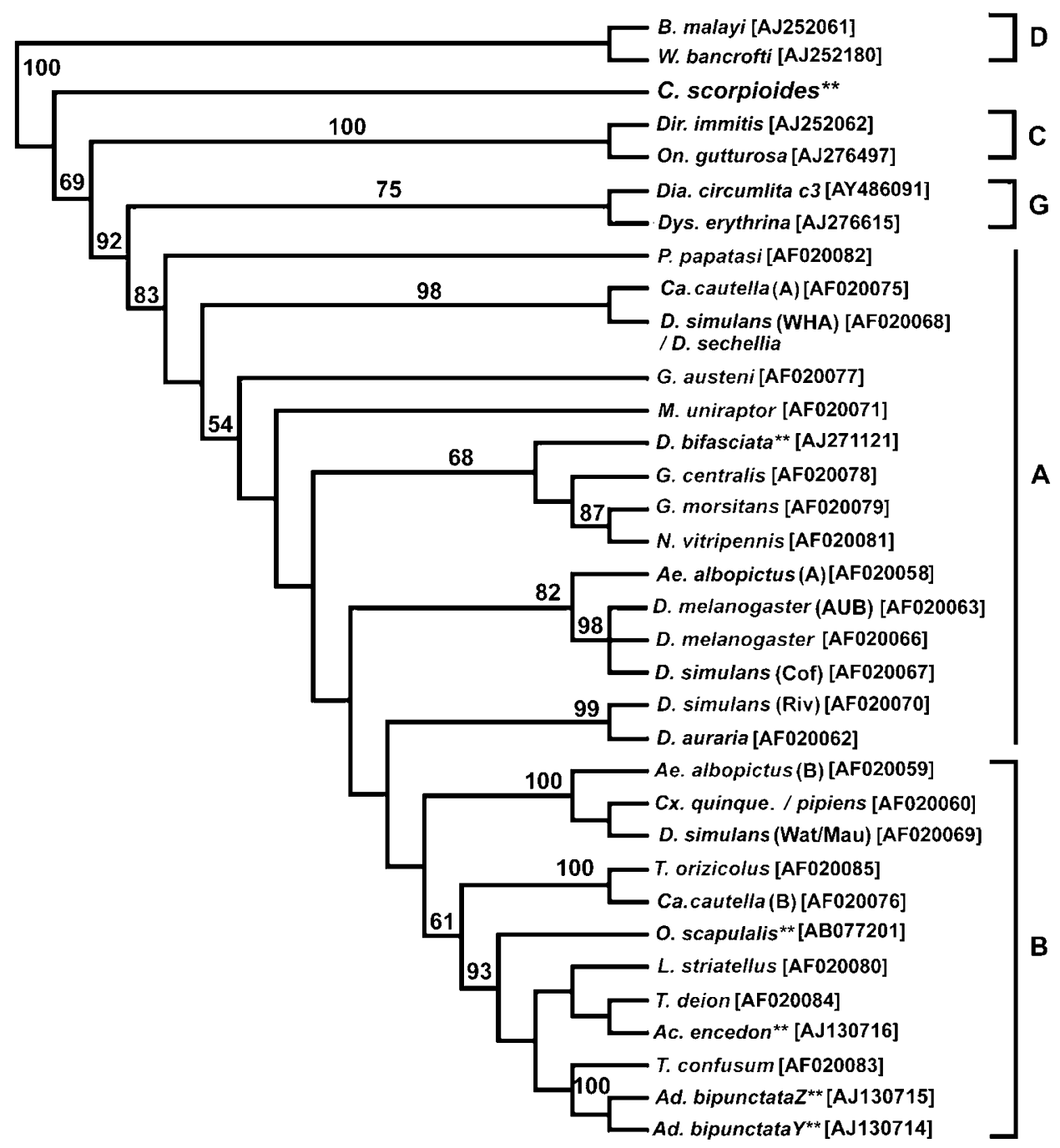

Figure 4 Maximum likelihood (ML) Wolbachia phylogenetic tree based on the addition to the alignment reported in Zhou et al (1998) of wsp gene sequence data from five male-killing Wolbachia strains, the strain infecting C. scorpioides, and two strains from each of the Wolbachia groups C, D and G. Wolbachia strains are identified by their host taxon name and GenBank accession number (in parentheses). Wolbachia groups are indicated (A-D and G). For the ML analysis, we used a GTR $+\mathrm{G}+\mathrm{I}$ substitution model (number of substitution types $=6$; nucleotide frequencies: $A=0.320 ; C=0.153 ; G=0.210 ; T=0.317$; proportion invariable sites $=0.326$ and gamma shape parameter $($ alpha $)=1.893$ ) selected by MODELTEST v3.6 (Posada and Crandall, 1998), and implemented in PAUP* (Swofford, 2002). Values above branches denote percent support for clades based on 100 bootstrap replicates. Wolbachia strains implicated in male killing are indicated by double asterisks.

harlequin beetle riding pseudoscorpion. In the FB line under investigation, sex ratio distortion exhibited a maternal line of descent and was associated with a high rate of spontaneous abortion and low reproductive success. Tetracycline treatment cured females of Wolbachia infection (as confirmed by negative PCR amplifications), significantly increased their reproductive success, and restored offspring sex ratio to 1:1. The higher reproductive success of cured females compared to that of infected females suggests that male killing, rather than feminization, is the mechanism responsible for the female bias. This interpretation is further supported by microscopic examination indicating that the broods carried by FB females frequently contained numerous embryos that had failed to develop normally (see Figure 5). It should be noted, however, that a feminizing bacterium could, in theory, be responsible, if it induced a substantial fecundity cost by incidentally killing a certain proportion of male embryos in the process of feminization. Such a bacterium would, in effect, be both a feminizer and a male killer. Alternatively, a feminizing bacterium could substantially reduce fecundity by incidentally killing both male and female embryos or by reducing the number of eggs ovulated. While theoretically possible, these mechanisms invoke multiple processes and therefore seem less parsimonious than the straightforward killing of male embryos.

Interestingly, the limited number of FB-line C. scorpioides males that survived to the adult stage nonetheless tested positive for the presence of Wolbachia. In Drosophila bifasciata, exposure of females to high temperature has been found to reduce, but not eliminate, Wolbachia from eggs. As a consequence, the proportion of males that survived to eclosion was shown to increase (Hurst et al, 


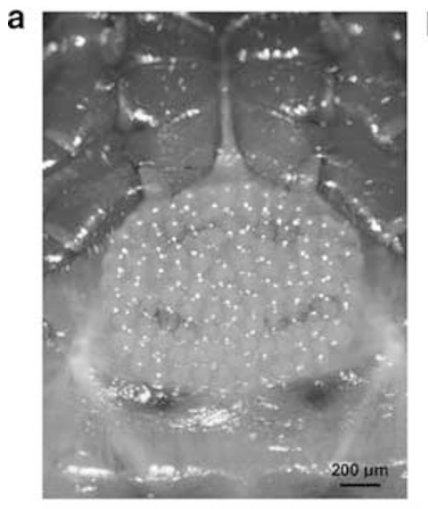

Normal brood

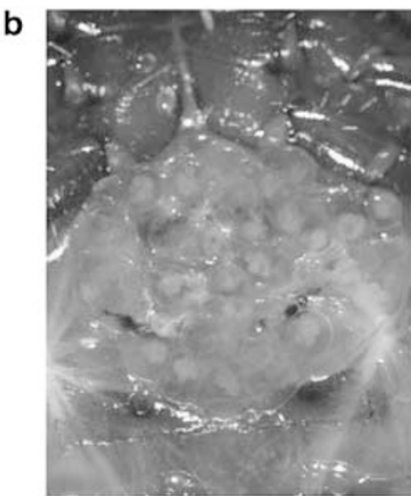

Wolbachia-infected brood
Figure 5 Early-stage development of C. scorpioides embryos in the brood sac produced by (a) an uninfected female, and (b) a Wolbachia-infected female. Compared to the uninfected female's closely-packed brood of embryos, the brood of the infected female contains many fewer developing embryos, with each embryo suspended in a visibly greater volume of nutritive fluid.

2000). Given the correlation between extent of female bias in a brood and the presence of detectable Wolbachia in protonymphs (Figure 3), it seems likely that a similar, bacterial-density-threshold effect operates in C. scorpioides due to variation in levels of Wolbachia transmission efficiency.

Our results provide circumstantial evidence for Wolbachia as the agent of sex ratio manipulation in $C$. scorpioides. However, tetracycline is a broad-spectrum antibiotic that is effective against not only Wolbachia but also a wide range of Gram-negative and Gram-positive bacteria. The presence of Wolbachia in the FB line therefore does not preclude the possibility that another tetracycline-sensitive microbe might be responsible for male killing in this pseudoscorpion. For example, the ladybird beetle, Adalia bipunctata, is known to harbour at least four different male-killing bacteria, including Rickettsia, Spiroplasma and two strains of Wolbachia (Schulenburg et al, 2002). The results of our sequencing study render it unlikely that the FB line is infected by more than a single Wolbachia strain, and our PCR assay with general eubacterial primers failed to detect other endosymbiotic bacteria. Moreover, the strong correlation between degree of female bias in a brood and the proportion of protonymphs testing positive for Wolbachia infection does suggest that natural variation in the efficacy of vertical transmission of Wolbachia is associated with variation in the level of male killing, pointing to Wolbachia as the most likely cause of sex ratio distortion. Nonetheless, a large-scale cloning and sequencing study (see Hurst et al, 1999b) would be required to determine unequivocally that sex ratio distortion is actually caused by this Wolbachia strain and not by the presence of another, as yet undetected, cellular endosymbiont.

If this newly reported Wolbachia strain is indeed responsible for male killing in C. scorpioides, it represents only the second reported case of a naturally occurring, male-killing strain outside of the Wolbachia group B clade, the first having been isolated from $D$. bifasciata and belonging to group A (Hurst et al, 2000). Other malekilling strains, isolated from the butterfly, Acraea encedon (Schulenburg et al, 2000), the moth, Ostrinia scapulalis,
Wolbachia in a pseudoscorpion

DW Zeh et al

(Kageyama et al, 2003) and the ladybird beetle, Adalia bipunctata (Hurst et al, 1999b), as well as from the flour beetle, Tribolium madens (Fialho and Stevens, 2000) are all members of group B. The large sequence divergence between the C. scorpioides strain and other male-killing Wolbachia strains is certainly consistent with the prediction that male killing is taxonomically more widespread than either feminization or parthenogenesis induction (Hurst et al, 1999b). It nonetheless remains to be determined whether the new strain belongs to a yet-tobe-discovered lineage of Wolbachia male killers or represents an isolated case of male-killing in its clade. Unfortunately, given the uniqueness of its sequence, the high level of homoplasy in the wsp- and ftsZ-based phylogenies, and the lack of comprehensive sampling from arachnids, the phylogenetic position of the $C$. scorpioides Wolbachia strain is uncertain. However, Wolbachia isolated from our FB line is currently being sequenced as part of a large-scale project, involving the sequencing of approximately 40 genes from some 50 Wolbachia strains (JH Werren, pers. comm.). Clarification of the phylogenetic position of the C. scorpioides strain should be forthcoming when the results of this study become available.

The discovery of male-killing in C. scorpioides is of particular interest because pseudoscorpions are viviparous (Weygoldt, 1969). Theory predicts that male killing should be favoured by kin selection, if the death of male embryos provides a fitness benefit to infected female siblings, thereby increasing fitness of the microbe (Werren, 1987; Hurst, 1991). For oviparous host species, it has been suggested that this fitness benefit may accrue through cannibalism, lowered risk of inbreeding or reduced sibling competition for environmental resources (Hurst, 1991). By contrast, in a live-bearing host such as C. scorpioides, reallocation of maternal resources (ovarian secretions) from dead male embryos to their female siblings provides a direct, physiological mechanism through which fitness compensation could favour the evolution of male killing by Wolbachia. Such reallocation could be particularly advantageous, if females typically ovulate more eggs than the number of embryos they are capable of carrying to term (Charlesworth, 1994), as is the case in C. scorpioides (JA Zeh, unpublished data). However, the data presented here for untreated FB-line females suggest that that there is limited scope for maternal resource reallocation to rescue female reproductive success. Certainly, in the early stages of development, female embryos of untreated mothers may have benefited from the death of their male siblings through increased availability of maternal nutrients (Figure 5). However, in most cases, this advantage was short-lived, since the majority of untreated FB females ultimately suffered spontaneous abortion of their entire broods.

While the cause of this high rate of spontaneous abortion remains to be determined, two mechanisms seem most plausible. First, because developing embryos actively draw maternal nutrients into the brood sac (Weygoldt, 1969), a 50\% reduction in the number of live embryos could reduce brood size to a level below the threshold necessary to maintain 'pregnancy.' Alternatively, the death of male embryos in a brood could result in maternal nutrients in the brood sac becoming contaminated with toxic decomposition products. Such toxins might interfere with the development of surviving 
embryos or could trigger an immune rejection response on the part of the mother. Regardless of mechanism, it seems likely that the susceptibility of Wolbachia-infected females to spontaneous abortion could be an important factor limiting the spread of the cellular endosymbiont and may be at least partially responsible for the relatively low frequency of male-killing in the harlequin beetle riding pseudoscorpion.

\section{Acknowledgements}

We are especially grateful for the logistical support provided by the Smithsonian Tropical Research Institute. We also thank La Autoridad Nacional del Ambiente (A.N.A.M.) for permission to collect in Panamá, Jack Werren for many useful discussions regarding the biology of Wolbachia, and three anonymous referees for constructive criticism. This research was funded by grants from the National Geographic Society (Grant 5333-94) and the US National Science Foundation (MCB0085335, DEB-0115555, IBN-0115986). MMB was supported by an NSF Research Experiences for Undergraduates award. Finally, for efficient and prompt processing of our sequencing samples, we thank Joan Rowe and Craig Osborne of The Nevada Genomics Center (NGC). The NGC is supported by NSF EPSCoR and NIH BRIN (P20 RR16464) Grants.

\section{References}

Altschul SF, Madden TL, Schäffer AA, Zhang J, Zhang Z, Miller $W$ et al (1997). Gapped BLAST and PSI-BLAST: a new generation of protein database search programs. Nucleic Acids Res 25: 3389-3402.

Bazzocchi C, Jamnongluk W, O'Neill SL, Anderson TJ, Genchi C, Bandi C (2000). wsp gene sequences from the Wolbachia of filarial nematodes. Curr Microbiol 41: 96-100.

Bouchon D, Rigaud T, Juchault P (1998). Evidence for widespread Wolbachia infection in isopod crustaceans: molecular identification and host feminization. Proc Roy Soc London B 265: 1081-1090.

Budar F, Touzet P, De Paepe R (2003). The nucleo-mitochondrial conflict in cytoplasmic male sterilities revisited. Genetica 117: 3-16.

Casiraghi M, Anderson TJ, Bandi C, Bazzocchi C, Genchi C (2001). A phylogenetic analysis of filarial nematodes: comparison with the phylogeny of Wolbachia endosymbionts. Parasitology 122: 93-103.

Charlat S, Hurst GD, Mercot H (2003). Evolutionary consequences of Wolbachia infections. Trends Genet 19: 217-223.

Charlesworth B (1994). The evolution of lethals in the $t$ haplotype system of the mouse. Proc Roy Soc London B 258: 101-107.

Cordaux R, Michel-Salzat A, Bouchon D (2001). Wolbachia infection in crustaceans: novel hosts and potential routes for horizontal transmission. J Evol Biol 14: 237-243.

Cosmides LM, Tooby J (1981). Cytoplasmic inheritance and intragenomic conflict. J Theor Biol 89: 83-129.

Dunn AM, Smith JE (2001). Microsporidian life cycles and diversity: the relationship between virulence and transmission. Microbes Infect 3: 381-388.

Dyson EA, Kamath MK, Hurst GDD (2002). Wolbachia infection associated with all-female broods in Hypolimnas bolina (Lepidoptera: Nymphalidae): evidence for horizontal transmission of a butterfly male killer. Heredity 88: 166-171.

Eberhard WG (1980). Evolutionary consequences of intracellular organelle competition. Q Rev Biol 55: 231-249.
Felsenstein J (1985). Confidence limits on phylogenies: an approach using the boostrap. Evolution 39: 783-791.

Fialho RF, Stevens L (2000). Male-killing Wolbachia in a flour beetle. Proc Roy Soc London B 267: 1469-1474.

Gherna RL, Werren JH, Weisburg W, Cote R, Woese CR, Mandelco L et al (1991). Arsenophonus nasoniae Gen-Nov, SpNov, the causative agent of the son-killer trait in the parasitic wasp Nasonia vitripennis. Int J Systematic Bacteriol 41: 563-565.

Hurst GDD, Bandi C, Sacchi L, Cochrane AG, Bertrand D, Karaca I et al (1999a). Adonia variegata (Coleoptera: Coccinellidae) bears maternally inherited Flavobacteria that kill males only. Parasitology 118: 125-134.

Hurst GDD, Jiggins FM, von der Schulenburg JHG, Bertrand D, West SA, Goriacheva II et al (1999b). Male-killing Wolbachia in two species of insect. Proc Roy Soc London B 266: 735-740.

Hurst GDD, Johnson AP, Schulenburg JHvd, Fuyama Y (2000). Male-killing Wolbachia in Drosophila: a temperature-sensitive trait with a threshold bacterial density. Genetics 156: 699-709.

Hurst LD (1991). The incidences and evolution of cytoplasmic male killers. Proc Roy Soc London B 244: 91-99.

Jeyaprakash A, Hoy MA (2000). Long PCR improves Wolbachia DNA amplification: wsp sequences found in $76 \%$ of sixtythree arthropod species. Insect Mol Biol 9: 393-405.

Jiggins FM, Hurst GDD, Jiggins CD, Schulenburg JHVD, Majerus MEN (2000). The butterfly Danaus chrysippus is infected by a male-killing Spiroplasma bacterium. Parasitology 120: 439-446.

Kageyama D, Nishimura G, Hoshizaki S, Ishikawa Y (2003). Two kinds of sex ratio distorters in a moth, Ostrinia scapulalis. Genome 46: 974-982.

Lawson ET, Mousseau TA, Klaper R, Hunter MD, Werren JH (2001). Rickettsia associated with male-killing in a buprestid beetle. Heredity 86: 497-505.

Lo N, Casiraghi M, Salati E, Bazzocchi C, Bandi C (2002). How many Wolbachia supergroups exist? Mol Biol Evol 19: 341-346.

Maddison DR, Maddison WP (2002). MacClade 4: Analysis of Phylogeny and Character Evolution, Version 4.05. Sinauer Associates: Sunderland, MA.

Majerus MEN (2003). Sex Wars: Genes, Bacteria, and Biased Sex Ratios. Princeton University Press: Princeton.

Makioka T (1968). Morphological and histochemical studies on embryos and ovaries during the embryo-breeding of the pseudoscorpions Garypus japonicus. Sci Rep Tokyo Kyoiku Daigaku Sec B 13: 207-227.

Newcomer SD, Zeh JA, Zeh DW (1999). Genetic benefits enhance the reproductive success of polyandrous females. Proc Natl Acad Sci USA 96: 10236-10241.

Posada D, Crandall KA (1998). MODELTEST: testing the model of DNA substitution. Bioinformatics 14: 817-818.

Rowley SM, Raven RJ, McGraw EA (2004). Wolbachia pipiensis in Australian spiders. Curr Microbiol 49: 208-214.

Schulenburg JHVD, Hurst GDD, Huigens GD, van Meer MM, Jiggins FM, Majerus ME (2000). Molecular evolution and phylogenetic utility of Wolbachia ftsZ and wsp gene sequences with special reference to the origin of male killing. Mol Biol Evol 17: 584-600.

Schulenburg JHVD, Hurst GDD, Tetzlaff D, Booth GE, Zakharov IA, Majerus ME (2002). History of infection with different male-killing bacteria in the two-spot ladybird beetle Adalia bipunctata revealed through mitochondrial DNA sequence analysis. Genetics 160: 1075-1086.

Stouthamer R, Kazmer DJ (1994). Cytogenetics of microbeassociated parthenogenesis and its consequences for gene flow in Tricogramma wasps. Heredity 73: 317-327.

Swofford DL (2002). PAUP*: Phylogenetic Analysis Using Parsimony (*And Other Methods), Version 4.0B10. Sinauer Associates: Sunderland.

Weisburg WG, Barns SM, Pelletier DA, Lane DJ (1991). 16s ribosomal DNA amplification for phylogenetic study. J Bacteriol 173: 697-703. 
Werren JH (1987). The coevolution of autosomal and cytoplasmic sex-ratio factors. I Theor Biol 124: 317334.

Werren JH (1997). Biology of Wolbachia. Ann Rev Entomol 42: 587-609.

Werren JH, Hurst GDD, Zheng W, Breeuwer JAJ, Stouthamer R, Majerus MEN (1994). Rickettsial relative associated with male killing in the ladybird beetle (Adalia bipunctata). J Bacteriol 176: 388-394.

Werren JH, Zhang W, Guo LR (1995). Evolution and phylogeny of Wolbachia: reproductive parasites of arthropods. Proc Roy Soc London B 261: 55-63.

Weygoldt P (1969). The Biology of Pseudoscorpions. Harvard University Press: Cambridge.

Williamson DL, Poulson DF (1979). Sex ratio organisms (spiroplasmas) of Drosophila. In: Whitcomb RF, Tully JG (eds) The Mycoplasmas: Plant and Insect Mycoplasmas. Academic Press: New York.

Zeh DW, Smith RL (1985). Paternal investment by terrestrial arthropods. Am Zool 25: 785-805.

Zeh DW, Zeh JA (1994). When morphology misleads interpopulation uniformity in sexual selection masks genetic divergence in harlequin beetle-riding pseudoscorpion populations. Evolution 48: 1168-1182.
Zeh DW, Zeh JA (2000). Reproductive mode and speciation: the viviparity-driven conflict hypothesis. BioEssays 22: 938-946.

Zeh DW, Zeh JA, Bermingham E (1997). Polyandrous, spermstoring females: carriers of male genotypes through episodes of adverse selection. Proc Roy Soc London B 264: 119-125.

Zeh DW, Zeh JA, Coffroth MA, Bermingham E (1992). Population-specific DNA fingerprints in a neotropical pseudoscorpion (Cordylochernes scorpioides). Heredity 69: 201-208.

Zeh DW, Zeh JA, May CA (1994). Charomid cloning vectors meet the pedipalpal chelae: single-locus minisatellite DNA probes for paternity assignment in the beetle-riding pseudoscorpion. Mol Ecol 3: 517-522.

Zeh JA, Newcomer SD, Zeh DW (1998). Polyandrous females discriminate against previous mates. Proc Natl Acad Sci USA 95: 13732-13736.

Zeh JA, Zeh DW (2001). Spontaneous abortion depresses female sexual receptivity in a viviparous arthropod. Anim Behav 62 427-433.

Zeh JA, Zeh DW, Bonilla MM (2003). Phylogeography of the harlequin beetle-riding pseudoscorpion and the rise of the Isthmus of Panamá. Mol Ecol 12: 2759-2769.

Zhou W, Rousset F, O'Neil S (1998). Phylogeny and PCR-based classification of Wolbachia strains using wsp gene sequences. Proc Roy Soc London B 265: 509-515. 